\title{
Surface Modifications during a Catalytic Reaction: a Combined APT and FIB/SEM Analysis of Surface Segregation.
}

\author{
Cédric Barroo ${ }^{1,2}$, Nare Janvelyan ${ }^{2}$, Branko Zugic ${ }^{2}$, Andrew P. Magyar ${ }^{3}$, Austin J. Akey ${ }^{3}$, Juergen
}

Biener $^{4}$, Cynthia M. Friend ${ }^{1,2}$, and David C. Bell ${ }^{1,3}$

1. John A. Paulson School of Engineering and Applied Sciences, Harvard University, Cambridge MA, USA

2. Department of Chemistry and Chemical Biology, Harvard University, Cambridge MA, USA

3. Center for Nanoscale Systems, Harvard University, Cambridge MA, USA

${ }^{4}$ Nanoscale Synthesis and Characterization Laboratory, Lawrence Livermore National Laboratory, Livermore CA, USA

To improve the understanding of catalytic processes, the surface structure and composition of the active materials need to be determined before and after reaction. Morphological changes may occur under reaction conditions and can dramatically influence the reactivity and/or selectivity of a catalyst. Goldbased catalysts with different architectures are currently being developed for selective oxidation reactions at low temperatures [1]. Specifically, nanoporous $\mathrm{Au}$ (npAu) with a composition of $\mathrm{Au}_{97}-\mathrm{Ag}_{3}$ is obtained by dealloying a $\mathrm{Ag}_{70}-\mathrm{Au}_{30}$ bulk alloy. Recent studies highlight the efficiency of $\mathrm{npAu}$ catalysts for methanol oxidation using ozone to activate the catalysts before methanol oxidation. In this work, we studied the morphological and compositional changes occurring at the surface of Au-based catalysts in certain conditions.

To do so, we first analysed nanoporous $\mathrm{Au}$ catalysts, with a composition of $\mathrm{Au}_{97}-\mathrm{Ag}_{3}$, by atom probe tomography (APT) to get better insight of the Ag distribution within the Au backbone. APT is a powerful technique to characterize the composition and 3D structure of materials at the atomic-scale. However, the presence of pores make the APT analysis and reconstruction difficult, thus new developments in sample preparation are required. Using a combination of in-situ e-beam-directed chemical vapor deposition in the focused ion beam (FIB) and lift-out techniques, we were able to successfully image npAu samples by atom probe tomography and analyse the segregation of Ag atoms in the npAu sample. From left to right, Figure 1 shows a schematic representation of npAu, a typical sample prepared for APT analysis (the pores have been filled with W) and the three-dimensional reconstruction of the sample; the latter suggests a non-uniform enrichment of $\mathrm{Ag}$ at the surfaces of nanoporous Au. Complimentary experiments were also performed on a bulk sample of the same composition using APT, FIB/SEM and X-Ray photoemission spectroscopy (XPS). This bulk sample was exposed to different treatments in a flow reactor: 1) thermal treatment: 1 hour at $150^{\circ} \mathrm{C}$ under flowing $\mathrm{He}-2$ ) ozone treatment: $150^{\circ} \mathrm{C}$ under a flow of $3 \% \mathrm{O}_{3}$ in $\mathrm{O}_{2}-\mathrm{He}$ with different exposure durations -3 ) reaction conditions: 1 hour at $150^{\circ} \mathrm{C}$ under a flow of $6.5 \% \mathrm{MeOH}$ and $20 \% \mathrm{O}_{2}-\mathrm{He}$. XPS and APT confirm the surface segregation of $\mathrm{Ag}$ (as silver oxide species) after ozone treatment, which is then reduced after exposing the catalyst to reaction conditions.

Further experiments were performed on a bulk $\mathrm{Ag}_{70}-\mathrm{Au}_{30}$ sample. The same treatments as the npAu sample were performed to follow changes during the different steps of the catalytic process. The ozone treatment induces the segregation of $\mathrm{Ag}$ at the surface, which forms a black layer composed mainly of $\mathrm{Ag}_{2} \mathrm{O}$. This oxide layer can be observed by a FIB/SEM cross-section as a distinct phase detached from the bulk sample. Removal of this oxide layer exposes the remaining sample with a composition of $\mathrm{Ag}_{56^{-}}$ 
$\mathrm{Au}_{44}$. Therefore, the surface segregation of $\mathrm{Ag}$ as a distinct layer is accompanied by Ag depletion of the bulk sample. The Ag-depleted region extends over a depth of a few $\mu \mathrm{m}$ (the depth depends on the duration of the ozone treatment). Energy-Dispersive X-ray spectroscopy (EDX) mapping of the crosssection indicates that the Ag-depleted layer is of homogeneous composition. The composition of the layer after reaction conditions is similar as after ozone treatment: reaction conditions don't affect the composition of the Ag-depleted region.

This study highlights the importance of ozone treatment in the segregation of Ag at the surface, which can dramatically influence the local chemistry and morphology of a catalyst. The combination of APT, FIB/SEM and XPS allows studying the surface and subsurface compositional and morphological changes of the sample after various physicochemical treatments, and also allows the segregation behaviour of Ag in different Au-based catalysts to be correlated. [2]

\section{References:}

[1] J. Biener, M. M. Biener, R. J. Madix and C. M. Friend. ACS Catal. 5 (2015) 6263

[2] This work was supported as part of the Integrated Mesoscale Architectures for Sustainable Catalysis - IMASC, an Energy Frontier Research Center funded by the U.S. Department of Energy, Office of Science, Basic Energy Sciences under Award \# DE-SC0012573. Work at LLNL was performed under the auspices of the U.S. Department of Energy by LLNL under Contract DE-AC52-07NA27344. C.B. acknowledges postdoctoral fellowships through the Belgian American Educational Foundation (BAEF) as well as Wallonie-Bruxelles International (Excellence grant WBI.WORLD) foundations.

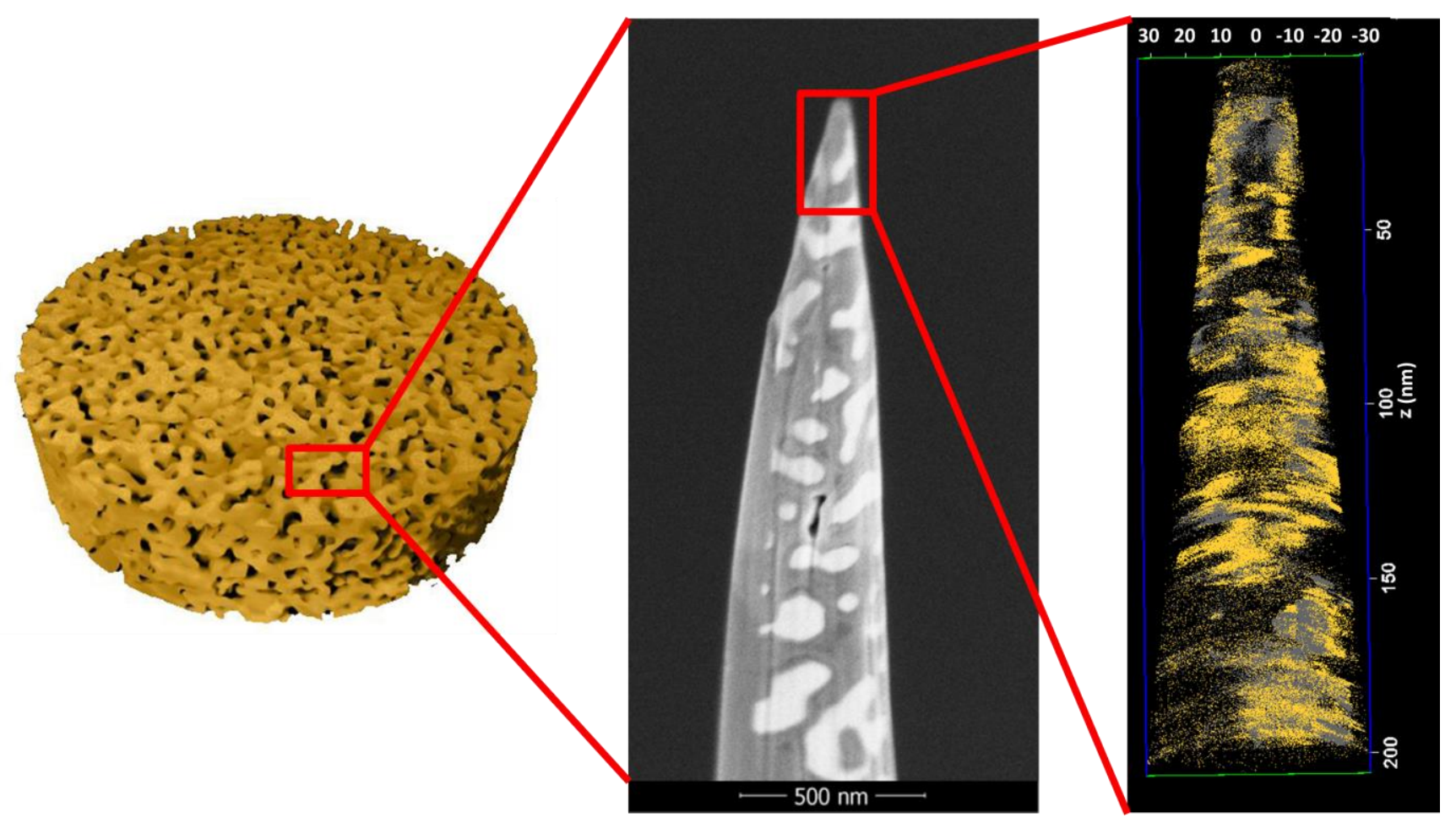

Figure 1. (left) Schematic representation of nanoporous gold (npAu) catalyst used for selective oxidation reaction - (middle) SEM image of an atom probe tip after filling the pores with $\mathrm{W}$ (Au ligaments appear brighter) - (right) 3D reconstruction of the APT sample showing the distribution of Ag atoms within the Au backbone 